\title{
Waveguide-integrated PdSe2 photodetector with high responsivity and speed in the near-infrared range across the $0+E+S+C$ bands
}

Jianghong Wu

Westlake University

Hui Ma

Zhejiang University

Chuyu Zhong

Zhejiang University

Maoliang Wei

Zhejiang university

Chunlei Sun

Westlake University

Yuting Ye

Westlake University

Yan Xu

Westlake University

Bo Tang

Institute of Microelectronics, Chinese Academy of Sciences

Ye Luo

Westlake University

Boshu Sun

Zhejiang university

Jialing Jian

Westlake University

Hao Dai

Zhejiang university

Hongtao Lin

Zhejiang University

Lan Li ( $\square$ lilan@westlake.edu.cn )

Westlake University https://orcid.org/0000-0002-9097-9157 
Keywords:

Posted Date: March 2nd, 2022

DOI: https://doi.org/10.21203/rs.3.rs-1399761/v1

License: (c) (1) This work is licensed under a Creative Commons Attribution 4.0 International License. Read Full License 


\section{Waveguide-integrated $\mathrm{PdSe}_{2}$ photodetector with high responsivity}

\section{and speed in the near-infrared range across the $\mathrm{O}+\mathrm{E}+\mathrm{S}+\mathrm{C}$ bands}

Jianghong $\mathrm{Wu}^{1,2,3 \#}$, Hui $\mathrm{Ma}^{1 \#}$, Chuyu Zhong ${ }^{1 \#}$, Maoliang $\mathrm{Wei}^{1}$, Chunlei Sun ${ }^{2,3}$, Yuting $\mathrm{Ye}^{2,3}$, Yan $\mathrm{Xu}^{2,3}$, Bo Tang ${ }^{4}$, Ye Luo ${ }^{2,3}$, Boshu Sun ${ }^{1}$, Jialing Jian ${ }^{2,3}$, Hao Dai ${ }^{1}$, Hongtao Lin ${ }^{1 *}$, and Lan $\mathrm{Li}^{2,3^{*}}$

${ }^{1}$ State Key Laboratory of Modern Optical Instrumentation, College of Information Science and Electronic Engineering, Zhejiang University, Hangzhou 310027, China. E-mail: hometown@zju.edu.cn

${ }^{2}$ Key Laboratory of 3D Micro/Nano Fabrication and Characterization of Zhejiang Province, School of Engineering, Westlake University, Hangzhou 310024, China. E-mail: lilan@westlake.edu.cn

${ }^{3}$ Institute of Advanced Technology, Westlake Institute for Advanced Study, 18 Shilongshan Road, Hangzhou 310024, China

${ }^{4}$ Institute of Microelectronics, Chinese Academic Society, Beijing 100029, China

\# J.W., H.M., and C.Z. contributed equally to this work.

\section{ABSTRACT}

Hybrid integration of two-dimensional (2D) materials on a silicon photonic platform enables diverse exploration of novel active functions and significant improvement in device performance for nextgeneration silicon integrated photonic circuits. 2D transition metal dichalcogenide materials (TMDCs) have attracted extensive attention for photodetectors, but developing high-performance waveguide-integrated photodetectors based on conventionally investigated 2D TMDCs at the telecom C-band is still a big challenge because of their large optical bandgap and slow carrier mobility. Here, we propose $\mathrm{PdSe}_{2}$, a new type of 2D TMDC, to be integrated with silicon photonic components for on-chip photodetection with high responsivity and speed in the datacom and telecom optical bands ranging from the O-band to the C-band. The obtained waveguide-integrated photodetectors show a high responsivity of $1190.2 \mathrm{~mA} \cdot \mathrm{W}^{-1}$ at $1550 \mathrm{~nm}$ and a low noise-equivalent power of $4.0 \mathrm{pW} \cdot \mathrm{Hz}^{-0.5}$ at $5 \mathrm{~V}$. The $3 \mathrm{~dB}$ bandwidth reaches up to $1.5 \mathrm{GHz}$, and the measured data rate is $2.5 \mathrm{Gbit} \cdot \mathrm{s}^{-1}$. The achieved $\mathrm{PdSe}_{2}$ photodetectors provide new insights to explore the integration of novel 2D TMDCs with silicon photonics and demonstrate the potential of integrated photodetectors for applications in diverse areas, including optical communications, on-chip spectroscopy, and sensing. 


\section{INTRODUCTION}

Over the past decade, two-dimensional (2D) materials have demonstrated novel and intriguing optoelectronic properties, including ultrafast mobility, broadband absorption, strong nonlinear response and dangling-bond-free surface, exhibiting great potential for both electronic and optoelectronic devices. ${ }^{1-10}$ Especially, integrating 2D materials with silicon integrated photonic circuits is a highly promising application. A plethora of waveguide-integrated 2D materials optoelectronic devices, including light sources, ${ }^{11,12}$ switches, ${ }^{13}$ polarizers,${ }^{14}$ modulators ${ }^{15,16}$ and photodetectors, ${ }^{17-19}$ have already been realized. Among these prototypical optoelectronic devices, waveguide-integrated photodetectors have attracted considerable attention, essential for multiple applications such as optical communications and interconnects, ${ }^{20,21}$ spectroscopy, ${ }^{22}$ sensing, ${ }^{23}$ imaging, ${ }^{24}$ and quantum computing. ${ }^{25}$ Among all 2D materials, graphene has been extensively studied for waveguide-integrated photodetectors, which in some aspects have shown competitive performance to Ge and III-V semiconductor counterparts. ${ }^{26-29}$ However, graphene photodetectors suffer from a large dark current because of a zero bandgap. Nowadays, 2D semiconductors such as black phosphorus (BP), $\mathrm{MoTe}_{2}$ and $\mathrm{Bi}_{2} \mathrm{O}_{2} \mathrm{Se}$ are alternative candidates for waveguide-integrated photodetectors at the current telecommunication bands. ${ }^{30-33}$ Actually, the low air-stability of BP limits the device fabrication and applications. A large optical bandgap for $\mathrm{MoTe}_{2}(1.04 \mathrm{eV})$ and $\mathrm{Bi}_{2} \mathrm{O}_{2} \mathrm{Se}(0.9 \mathrm{eV})$ prevents it from optical detection at the C-band.

Recently, 2D PdSe 2 flake with great air-stability has been a new candidate for optoelectronic applications at near- and mid-IR wavelengths. ${ }^{34-36} \mathrm{PdSe}_{2}$ flakes possess a large bandgap tunability, ranging from $0.03 \mathrm{eV}$ (bulk) to $\sim 1.3 \mathrm{eV}$ (monolayer). ${ }^{37,38}$ The experimental effective electron mobility of few-layer $\mathrm{PdSe}_{2}$ is more than $200 \mathrm{~cm}{ }^{2} \cdot \mathrm{V}^{-1} \cdot \mathrm{s}^{-1} \cdot{ }^{39}$ Moreover, a wafer-scale $\mathrm{PdSe}_{2}$ film can be obtained by a simple selenization process of pre-deposited Pd film, ${ }^{40,41}$ and centimeter-scale $\mathrm{PdSe}_{2}$ film (thinner than $10 \mathrm{~nm}$ ) can be prepared by Chemical Vapor Deposition method, ${ }^{42}$ which ensures the fabrication of a device array on a large scale. To date, free-space $\mathrm{PdSe}_{2}$ photodetectors have already been investigated. For example, a $\mathrm{PdSe}_{2}$ phototransistor showed photoresponse ranging from the visible to mid-infrared wavelengths up to $4.05 \mu \mathrm{m}$. The responsivity was 708 $\mathrm{A} \cdot \mathrm{W}^{-1}$ under a low incident power at $1064 \mathrm{~nm},{ }^{43}$ and this high responsivity mainly resulted from the trap state, which brought about a slow dynamic response as well. A photodiode based $\mathrm{PdSe}_{2} / \mathrm{Si}$ heterostructure possessed a responsivity of $300.2 \mathrm{~mA} \cdot \mathrm{W}^{-1}$ at $780 \mathrm{~nm}$, and a small $3 \mathrm{~dB}$ bandwidth 
of $30 \mathrm{kHz}$ limited it for high-speed applications. ${ }^{41}$ Moreover, the van der Waals heterostructure such as $\mathrm{InSe} / \mathrm{PdSe}_{2}$ and $\mathrm{PdSe}_{2} / \mathrm{MoS}_{2}$ heterostructure showed a responsivity of $58.8 \mathrm{~A} \cdot \mathrm{W}^{-1}$ at $1650 \mathrm{~nm}$, and $42.1 \mathrm{~A} \cdot \mathrm{W}^{-1}$ at $10.6 \mu \mathrm{m}$, respectively, but the rise/fall times of these photodetectors were tens of millisecond. ${ }^{44,45} \mathrm{Up}$ to now, realizing a $\mathrm{PdSe}_{2}$ photodetector with high responsivity and large bandwidth is still a challenge and highly desired. Indeed, a waveguide architecture is attractive for designing photodetectors with both high responsivity and large bandwidth. It can simultaneously ensure adequate absorption via evanescent field coupling over a long propagation distance and decrease the transit time of photoinduced carriers with a short channel.

In this work, we propose and demonstrate waveguide-integrated $\mathrm{PdSe}_{2}$ photodetectors operating in the datacom and telecom optical bands ranging from the O-band to the C-band, for the first time, with both high responsivity and large bandwidth. The significant optoelectronic properties of multilayer $\mathrm{PdSe}_{2}$ film, including anisotropy and ultrafast carrier dynamics, are characterized. We integrate silicon-on-insulator (SOI) ridge waveguides with the multilayer $\mathrm{PdSe}_{2}$ flakes, which were exfoliated from bulk materials and transferred by an imprint-transfer process. Such devices ensure sufficient optical absorption for the fundamental mode of TE polarization $\left(\mathrm{TE}_{0}\right)$ and a low mode mismatching loss. The present $\mathrm{PdSe}_{2}$ waveguide-integrated photodetectors possess a broadband spectral operation, a high responsivity of $1190.2 \mathrm{~mA} \cdot \mathrm{W}^{-1}$, and a low noise-equivalent power (NEP) of $4.0 \mathrm{pW} \cdot \mathrm{Hz}^{-0.5}$ at $5 \mathrm{~V}$ at $1550 \mathrm{~nm}$. Additionally, a $3 \mathrm{~dB}$ bandwidth reaches up to $1.5 \mathrm{GHz}$, and the receiver eye diagram indicates $\mathrm{PdSe}_{2}$ photodetector could realize a data transmission rate being more than $2.5 \mathrm{Gbit}^{-1} \mathrm{~s}^{-1}$. 
a

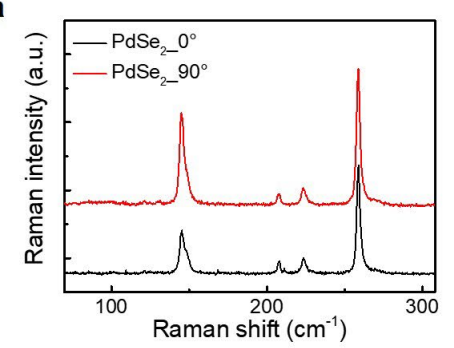

d

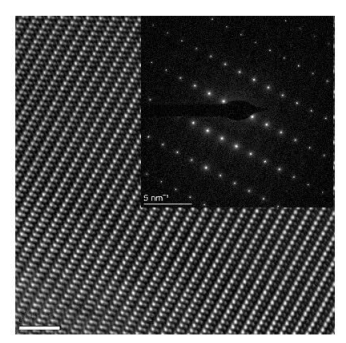

b

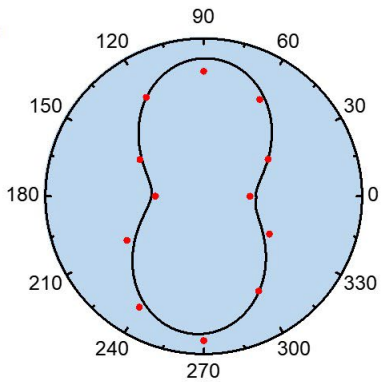

e

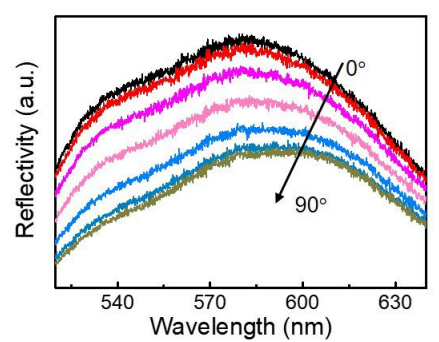

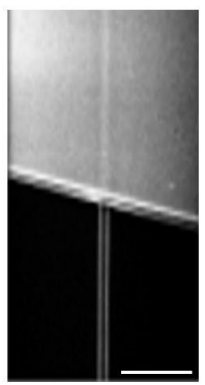

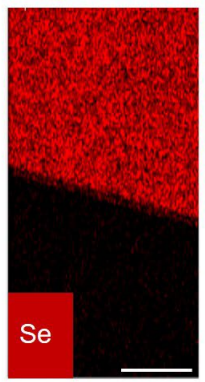

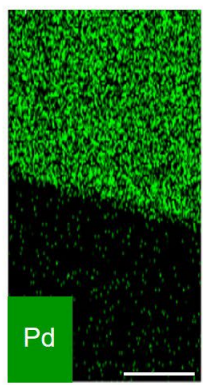

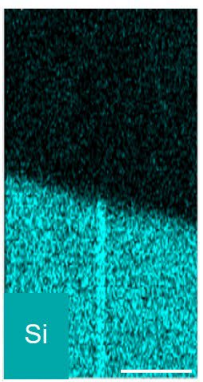

Fig. 1 Multilayer $\mathrm{PdSe}_{2}$ flake characterization. a Raman spectra of a multilayer $\mathrm{PdSe}_{2}$ flake excited by incident light at $532 \mathrm{~nm}$ under two different polarization states. b Polar plots of the measured and fitted peak intensities of the $A_{g}^{1}$ mode. c The energy-dispersive X-ray spectroscopy (EDS) mappings of a multilayer PdSe $\mathrm{Pl}_{2}$ flake on an SOI ridge waveguide. Scale bar: $5 \mu \mathrm{m}$. d Highresolution transmission electron microscope (TEM) image of a multilayer $\mathrm{PdSe}_{2}$ film. The illustration is the corresponding selected area electron diffraction (SAED) pattern. Scale bar: $2 \mathrm{~nm}$. e Reflection spectra of a multilayer $\mathrm{PdSe}_{2}$ flake with a white light source under different polarization states.

\section{RESULTS}

$\mathrm{PdSe}_{2}$ characterization. The lattice structure of the 2D-layer $\mathrm{PdSe}_{2}$ flake is a puckered pentagon, where the Pd atom is connected with four Se atoms through a covalent bond, constituting an asymmetric lattice structure, ${ }^{37}$ and the van der Waals force is dominant in the interactions between adjacent layers. Raman spectra of a multilayer PdSe $\mathrm{Plake}_{2}$ (purchased from Taizhou SUNANO New Energy CO., Ltd.) on $\mathrm{SiO}_{2}$ substrate excited by the $532 \mathrm{~nm}$ laser are shown in Fig. 1a, where four distinct Raman peaks at about 145, 208, 223, and $258 \mathrm{~cm}^{-1}$ are observed, which are corresponding to $A_{g}^{1}-B_{1 g}^{1}, A_{g}^{2}, B_{1 g}^{1}$, and $A_{g}^{3}$ modes, respectively. Furthermore, the measured Raman spectra under different polarization states are shown in Supplementary Fig. 1a, indicating the strong inplane anisotropic phonon vibration. The polarization-dependent Raman intensity of $A_{g}^{1}-B_{1 g}^{1}$ (Fig.1b) shows clear periodical variations of $\pi$. The chemical composition of a multilayer $\mathrm{PdSe}_{2}$ flake was characterized by X-ray photoelectron spectroscopy (XPS) measurement (Supplementary 
Figs. $1 \mathrm{~b}, \mathrm{c}$ ) in which four typical peaks at $54.6,55.4,336.7$, and $342.1 \mathrm{eV}$ are ascribed to $\mathrm{Se} 3 \mathrm{~d}_{5 / 2}$, $3 d_{3 / 2}, P d 3 d_{5 / 2}$, and $3 d_{3 / 2}$ orbitals, respectively. The energy-dispersive X-ray spectroscopy (EDS) mappings of a multilayer $\mathrm{PdSe}_{2}$ flake on an SOI ridge waveguide are shown in Fig. 1c, where Pd and Se elements show a uniform distribution. Moreover, the high-magnification TEM image and SAED pattern in Fig. 1d demonstrates the single-crystal characteristic of the exfoliated multilayer $\mathrm{PdSe}_{2}$ flake. Additionally, anisotropic optical properties were confirmed by the variation of the reflection spectra under different polarization states (Fig. 1e), and the reflectivity rises gradually when the polarization angle increases from $0^{\circ}$ to $90^{\circ}$, which indicates that the crystal orientation has an important impact on photon-to-electron transition.

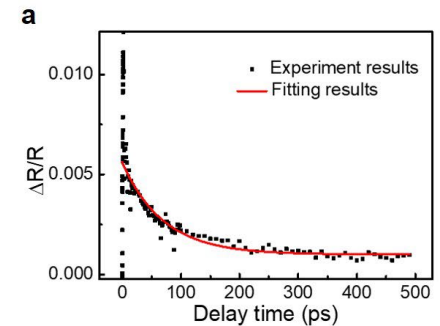

d

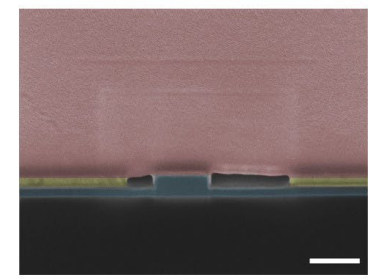

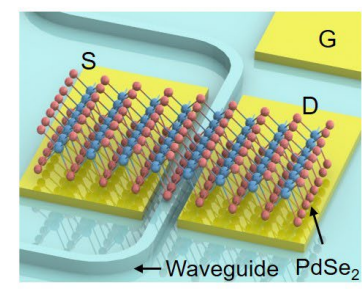

e

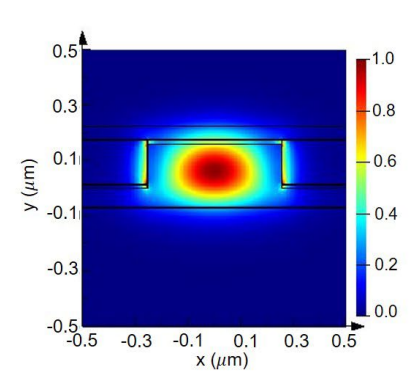

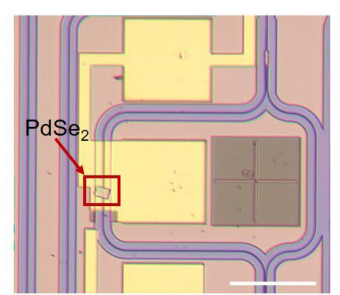

f

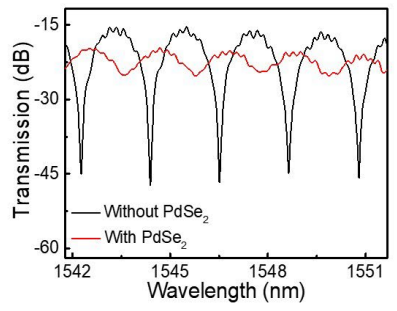

Fig. $2 \mathrm{PdSe}_{2}$ photodetector integrated with a silicon photonic circuit. a The measured ultrafast transient reflectance of a multilayer PdSe $\mathrm{Plake}_{2}$ (12 nm thick). b Three-dimensional illustration of the waveguide-integrated $\mathrm{PdSe}_{2}$ photodetector. c Optical image of a $\mathrm{PdSe}_{2}$ waveguide-integrated photodetector. Scale bar: $100 \mu \mathrm{m}$. d Cross-section scanning electron microscope (SEM) image of a $\mathrm{PdSe}_{2}$ photodetector. Scale bar: $500 \mathrm{~nm}$. e Calculated electric field profiles of the $\mathrm{TE}_{0}$ mode in the hybrid silicon/PdSe $\mathrm{Pa}_{2}$ waveguide, the thickness of the $\mathrm{PdSe}_{2}$ flake is $50 \mathrm{~nm}$. f The transmission spectra of the unbalanced MZI with and without a $\mathrm{PdSe}_{2}$ flake (155 nm thick).

The lifetime of photoinduced carriers was inferred by the ultrafast transient reflectance (TR) spectroscopy. Details of measurement setup could be found in the previous report. ${ }^{46} \mathrm{We}$ excited a multilayer $\mathrm{PdSe}_{2}$ flake with a $2.07 \mathrm{eV}$ pump pulse (50 fs pulse duration) and measured the reflectance change of this $\mathrm{PdSe}_{2}$ flake using a white-light continuum probe. The TR kinetics (Fig. 
2a) indicates that the lifetime of photoinduced carriers of this $\mathrm{PdSe}_{2}$ flake (12 nm thickness in Supplementary Fig. 1d) is about 68 ps. With the increase in $\mathrm{PdSe}_{2}$ thickness, a narrowing bandgap induces a shorter lifetime of photoinduced carriers, and the measured lifetime under $1550 \mathrm{~nm}$ wavelength illumination is $22 \mathrm{ps}$ for a multilayer $\mathrm{PdSe}_{2}$ flake (20 nm thick) in a previous report. ${ }^{47}$ The lifetime is longer than that counterpart of graphene, ${ }^{48}$ indicating $\mathrm{PdSe}_{2}$ photodetectors can not only realize a high photoconductive gain $\left(\mathrm{G}=\tau_{\text {life }} / \tau_{\text {tran }}, \tau_{\text {tran }}\right.$ is the drift transit time), but also a large $3 \mathrm{~dB}$ bandwidth.

Device concept and simulation. Figure $2 \mathrm{~b}$ shows the schematic configuration of the waveguideintegrated $\mathrm{PdSe}_{2}$ photodetector, where SOI ridge waveguide (220 nm thick) with an etching height of $150 \mathrm{~nm}$ is applied. The silicon waveguide was lightly doped, and the heavily doped area $\left(2 \times 10^{20}\right.$ $\mathrm{cm}^{-3}$ ) was close to the waveguide at a suitable distance, which can not only suppress unnecessary optical loss induced by the plasma dispersion effect, but also decrease the total resistance to optimize the device performance. A layer of $\mathrm{Al}_{2} \mathrm{O}_{3}(15 \mathrm{~nm}$ thick) was deposited by atomic layer deposition to avoid photoinduced carriers transferring between a $\mathrm{PdSe}_{2}$ flake and the bottom $\mathrm{Si}$ waveguide. Two Ti/Au pads $(5 / 100 \mathrm{~nm})$ were deposited, locating around the straight waveguide with a suitable distance, which effectively promoted the collection of photoinduced carriers and significantly avoided an unnecessary optical loss. A PdSe $e_{2}$ flake was transferred to the top of a waveguide, which connected these two Au pads. Incident light was coupled into/out waveguide by two grating couplers whose SEM images are illustrated in Supplementary Fig. 2. A typical optical image of the waveguide-integrated $\mathrm{PdSe}_{2}$ photodetector is shown in Fig. 2c, where a $\mathrm{PdSe}_{2}$ flake is on one arm of an unbalanced Mach-Zehnder interferometer (MZI). We integrated $\mathrm{PdSe}_{2}$ flakes with straight waveguides as well. The cross-section of a waveguide-integrated $\mathrm{PdSe}_{2}$ photodetector (Fig. 2d) indicates that an altitude difference remains at the top of the waveguide core, slab and Au pads, and a part of $\mathrm{PdSe}_{2}$ flake is suspended. The adhesion between a $\mathrm{PdSe}_{2}$ flake and the waveguide substrate was strong enough, which was approved by the photoresist coating.

Optical absorption of a $\mathrm{PdSe}_{2}$ flake is a significant factor for the photon-to-electron transition, thereby the simulated electric field profiles of the $\mathrm{TE}_{0}$ mode is illustrated (Fig. 2e). The simulation result indicates that a part of the electric field profiles of the $\mathrm{TE}_{0}$ mode is in the $\mathrm{PdSe}_{2}$ flake in a $\mathrm{PdSe}_{2} / \mathrm{Si}$ hybrid waveguide. The measured optical absorption of a $\mathrm{PdSe}_{2}$ flake was characterized by comparing the transmission spectra of an unbalanced MZI (Fig. 2f) with/without a $\mathrm{PdSe}_{2}$ flake. In 
this unbalanced MZI, beam splitter and combiner are realized by two $1 \times 2$ MMIs. The detailed performance of a $1 \times 2 \mathrm{MMI}$ is characterized by the serially cascaded MMIs, and the transmission spectra indicate a $1 \times 2 \mathrm{MMI}$ in our design could split incident light power equally (Supplementary Fig. 3). According to the change of extinction ratio (ER), the absorption coefficient of the $\mathrm{PdSe}_{2}$ ( $155 \mathrm{~nm}$ thick)/Si hybrid waveguide is calculated to be $0.14 \mathrm{~dB} / \mu \mathrm{m}$. We also demonstrated the transmission spectra variation of a straight waveguide with/without a $\mathrm{PdSe}_{2}$ flake (Supplementary Fig. 7a). Actually, the slight difference of grating coupler during different operations remains, leading to an experimental error of absorption coefficient of $\mathrm{PdSe}_{2} / \mathrm{Si}$ hybrid waveguide, which could be avoided by measuring from an MZI.

(a)

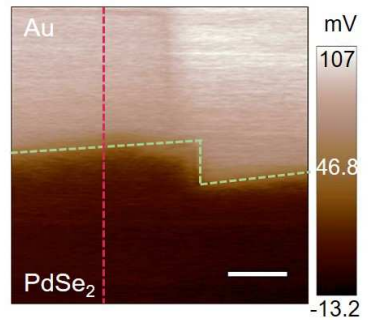

(d)

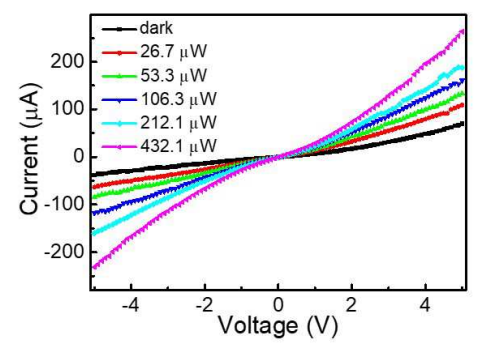

(b)

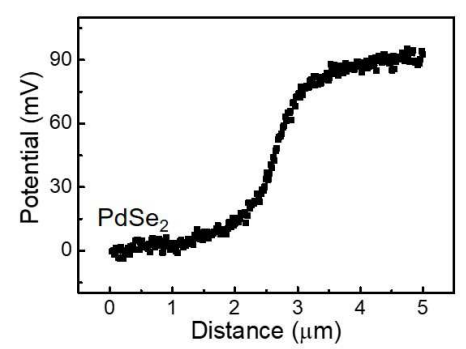

(e)

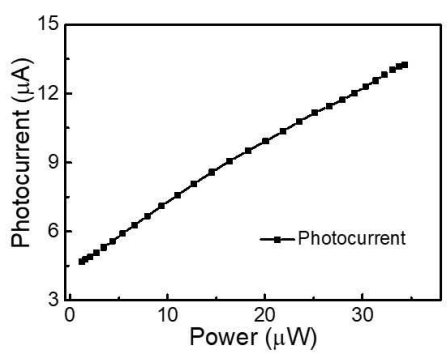

(c)

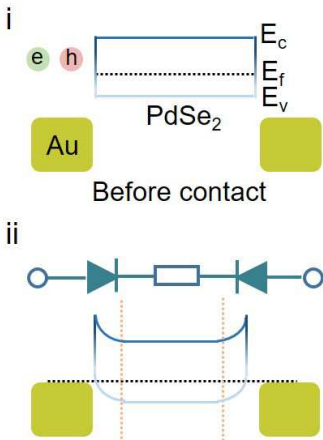

After contact

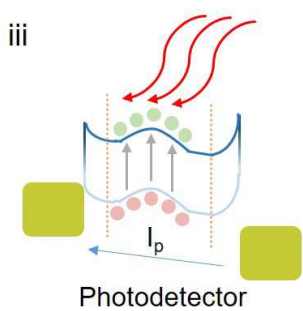

Fig. 3 Electronic characteristics of $\mathbf{P d S e}_{2}$ photodetector. a Kelvin probe force microscopy (KPFM) image of the Au-PdSe $e_{2}$ contact on a $\mathrm{Si} / \mathrm{SiO}_{2}$ substrate. Scale bar: $1 \mu \mathrm{m}$. b KPFM profile along with the red dashed line in Fig. a. $\mathbf{c}$ The energy band diagram of Au-PdSe $e_{2}-\mathrm{Au}$ junction at equilibrium, under illumination, at a bias voltage, and corresponding equivalent circuit. d Currentvoltage (IV) curves a waveguide-integrated $\mathrm{PdSe}_{2}$ photodetector (Sample 1, S1) in the dark and under illumination with different optical power at $1550 \mathrm{~nm}$. e Photocurrent versus incident optical power at $2 \mathrm{~V}$.

Electrical characterization. Next, we measured the contact potential difference $\left(\mathrm{V}_{\mathrm{CPD}}\right)$ between an $\mathrm{Au}$ electrode and a $\mathrm{PdSe}_{2}$ flake by Kelvin probe force microscopy (KPFM). The $\mathrm{V}_{\mathrm{CPD}}$ is defined as 
the difference of the work function between the probe tip and the measured sample, which can be expressed as $\mathrm{V}_{\mathrm{CPD}}=\left(\mathrm{W}_{\text {tip }}-\mathrm{W}_{\text {sample }}\right) /-\mathrm{e}$, where $\mathrm{W}_{\text {tip }}$ and $\mathrm{W}_{\text {sample }}$ are the work function of the probe tip and the sample, respectively. The KPFM image of a $\mathrm{PdSe}_{2}$-Au junction (Fig. 3a) indicates the different work functions for an $\mathrm{Au}$ electrode and a $\mathrm{PdSe}_{2}$ flake, where the green dot line is the edge of the $\mathrm{PdSe}_{2}$ flake and Au electrode. The detailed surface potential profile along the red dot line is illustrated in Fig. 3b, in which we can observe that the work function difference between the $\mathrm{Au}$ electrode and $\mathrm{PdSe}_{2}$ flake is about $90 \mathrm{meV}$. The smaller work function results in electron transferring from $\mathrm{PdSe}_{2}$ flake to Au electrode, the energy band sloping upward, and a built-in electric field coming into being at the contact interface (Fig. 3c). Therefore, the equivalent circuit of a realized $\mathrm{PdSe}_{2}$ photodetector can be regarded as a back-to-back Schottky junction (Fig. 3c) which brings about a small dark current. The photovoltaic effect (PVE) is usually dominant for free-space photodetectors based on $\mathrm{Au}$-semiconductor-Au structure, and photocurrent can be generated at the junction without an external bias voltage ${ }^{49}$ However, the optical absorption of a $\mathrm{PdSe}_{2}$ flake in our photodetector is mainly located on top of a waveguide surface (Fig. 2e), and the distance between the Au electrode and optical absorption area (the edge of the waveguide) is larger than the width of depletion area, thereby PVE is not the dominant mechanism for the present photodetector. Moreover, the photo-thermoelectric effect (PTE) probably contributes to the total photocurrent in a suspended device structure, but photocurrent is extremely weak at zero bias voltage. Thereby, PTE effect does not contribute to a photon-to-electron transition in the obtained $\mathrm{PdSe}_{2}$ photodetectors.

The static characteristics of a $\mathrm{PdSe}_{2}$ photodetector (Sample1) were measured, and IV curves in the dark and under illumination at $1550 \mathrm{~nm}$ were demonstrated (Fig. 3d). The nonlinear property of IV curves is attributed to a Schottky barrier at the contact interface between $\mathrm{Au}$ and $\mathrm{PdSe}_{2}$. Two primary aspects resulted in the Schottky contact. On the one hand, a low background doping level of the multilayer $\mathrm{PdSe}_{2}$ flake ${ }^{50}$ brings about a large depletion layer width and small thermionic and tunneling currents. On the other hand, a multilayer $\mathrm{PdSe}_{2}$ flake was transferred onto the Au electrode, avoiding unnecessary chemical disorder and Fermi-level pinning, leading to a better Schottky junction. ${ }^{51}$ The dark currents are $-38 \mu \mathrm{A}$ at $-5 \mathrm{~V}$, and the thickness of this $\mathrm{PdSe}_{2}$ flake is $171 \mathrm{~nm}$ (Supplementary Fig. 4), respectively. Moreover, a smaller dark current can be obtained by decreasing the thickness of the $\mathrm{PdSe}_{2}$ flake because of the increase of resistance. ${ }^{52}$ Furthermore, aligning the crystal orientation has an impact on total resistance. ${ }^{53}$ The anisotropic electronic 
transport (Supplementary Fig. 5) indicates resistance variation remains in multilayer $\mathrm{PdSe}_{2}$ flakes, which provides flexibility to device design. Current rises gradually (Fig. 3d) with the improving incident power, indicating that $\mathrm{PdSe}_{2}$ could be used for optical detection at C-band. Such a positive relationship between photocurrent and bias voltage is observed, and photocurrent at zero bias voltage is weak, illustrating photoconductive effect is the dominant mechanism for photocurrent. The photocurrent-incident optical power curve (Fig. 3e) at $2 \mathrm{~V}$ indicates the $\mathrm{PdSe}_{2}$ photodetector possesses a great linear relationship.

Responsivity varies as a function of bias voltage (Fig. 4a), because the higher efficiency of the generation-separation-collection process of photoinduced carriers can be realized at a larger bias voltage. The measured external responsivity $\left(\mathrm{R}=I_{p} / P_{\text {in }}\right)$ is $1190.2,285.7$, and $191.2 \mathrm{~mA} \cdot \mathrm{W}^{-1}$ at 5 $\mathrm{V}$ and $1550 \mathrm{~nm}$ for $\mathrm{S} 1, \mathrm{~S} 2$, and $\mathrm{S} 3$, respectively, and the external quantum efficiency (EQE=1.24× $\mathrm{R} / \lambda$ ) is $95.2 \%, 22.9 \%$, and $15.3 \%$ at $5 \mathrm{~V}$ for $\mathrm{S} 1, \mathrm{~S} 2$, and $\mathrm{S} 3$, respectively, where $\lambda$ is the wavelength of incident light. Such an external responsivity and EQE are higher than the counterparts based on graphene, ${ }^{54,55} \mathrm{BP},{ }^{32}$ and $\mathrm{MoTe}_{2} \cdot{ }^{56}$ The variation of responsivity for these three samples $(\mathrm{S} 1, \mathrm{~S} 2$, and S3) is mainly attributed to the thickness, length, and optical anisotropy of the multilayer $\mathrm{PdSe}_{2}$ flakes. The thickness of $\mathrm{PdSe}_{2}$ flakes in these three samples is 171, 186 and $284 \mathrm{~nm}$, respectively, which is characterized by the atomic force microscope (Supplementary Fig. 4). Moreover, the reflectivity varies under different polarization states (Fig. 1e), illustrating that optical anisotropy has a severe impact on optical absorption and photo-to-electron transition, which is consistent with previous reports. ${ }^{57,58}$

We then measured the channel current in the dark and under illumination with a back-gate voltage (Fig. $4 b$ ). $I_{d}-V_{g}$ curve in the dark shows a small on-off ratio of about 3 for a $\mathrm{PdSe}_{2}$ photodetector (S1), indicating that the $\mathrm{PdSe}_{2}$ flake in our experiment is $\mathrm{p}$-doped, which is consistent with previous reports. ${ }^{50}$ The thickness of the $\mathrm{PdSe}_{2}$ flake (171 nm thick) in our device is significantly greater than the Debye screening length, resulting in the device not being fully turned off, and a moderate gate-dependent transport. Two $\mathrm{PdSe}_{2}$ field-effect transistors (FET) on a $\mathrm{SiO} 2 / \mathrm{Si}$ substrate also verify the gate-dependent transport, one $\mathrm{PdSe}_{2} \mathrm{FET}$ (about $25 \mathrm{~nm}$ thick) demonstrates an ambipolar transport with an on-off ratio of about 11, and another $\mathrm{PdSe}_{2}$ FET (about $120 \mathrm{~nm}$ thick) shows the unipolar transport (Supplementary Fig. 6). The transport indicates that multilayer $\mathrm{PdSe}_{2}$ flake possesses a low background doping, because the onset of different doping locates about $\mathrm{V}_{\mathrm{g}}=0$ 
V. Moreover, the slight gate-dependent photocurrent is observed as well (Fig. 4b), which could be primarily attributed to the variation of optical absorption of $\mathrm{PdSe}_{2}$ flakes, but not the larger thermionic and tunneling currents, ${ }^{59}$ being similar with photodetectors based on graphene. ${ }^{60}$ As shown in Fig. 2d, carrier variation induced by a back-gate voltage mainly concentrates on the area at the top surface of a waveguide, thereby a back-gate voltage has little impact on the $\mathrm{PdSe}_{2}-\mathrm{Au}$ Schottky barriers, as well as thermionic and tunneling currents. Similar results, including the unipolar dark current and maximal photocurrent at $\mathrm{V}_{\mathrm{g}}=0 \mathrm{~V}$ were observed in another waveguideintegrated photodetector as well (Supplementary Fig. 7).

(a)

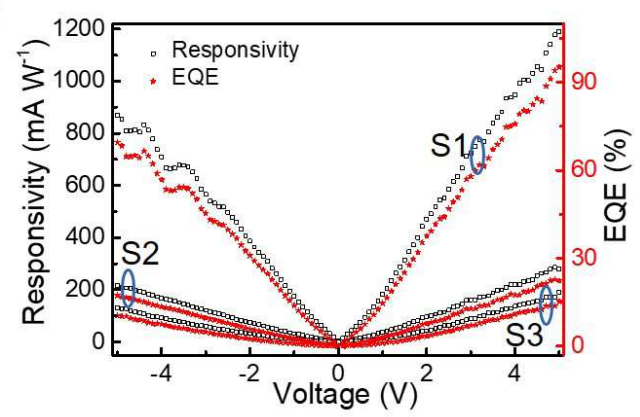

(c)

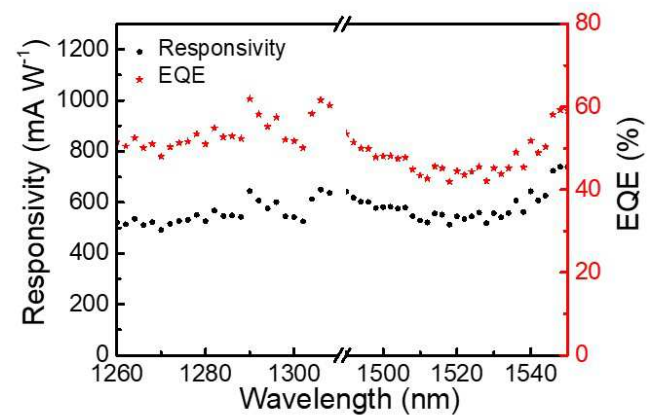

(b)

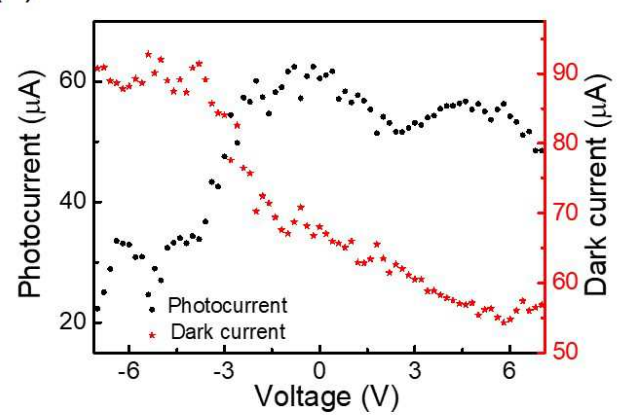

(d)

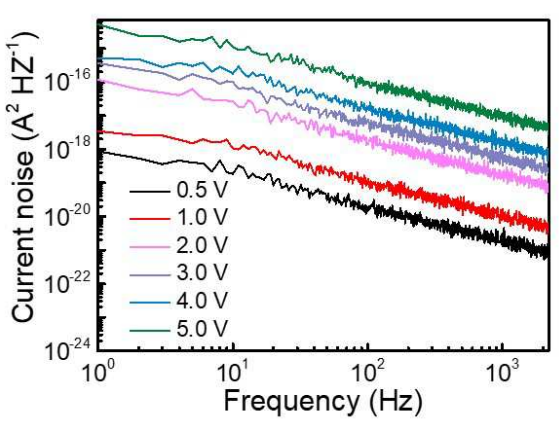

Fig. 4 Static performance of $\mathrm{PdSe}_{2}$ photodetectors. a Responsivity and EQE for S1, S2 and S3 at different bias voltage under the illustration of $1550 \mathrm{~nm}$. b Gate-tunable transport and photoresponse of a $\mathrm{PdSe}_{2}$ photodetector (S1). c Spectral response of $\mathrm{PdSe}_{2}$ photodetectors, the measured results at O-band are obtained by S4 and others are gotten by S1. d 1/f noise spectra of a waveguide-integrated $\mathrm{PdSe}_{2}$ photodetector (S1) under different applied voltages.

Spectral response. The spectral photoresponse for $\mathrm{PdSe}_{2}$ photodetectors at the current optical communication wavelengths was characterized in Fig. 4c, in which $\mathrm{PdSe}_{2}$ photodetectors demonstrated the broadband operation, and the responsivity was more than $500 \mathrm{~mA} \cdot \mathrm{W}^{-1}$ with input optical power being larger than $200 \mu \mathrm{W}$ at $5 \mathrm{~V}$. Limited by a finite optical bandwidth of grating couplers, we fabricated two photodetectors (S1 and S4) to show this broadband spectral 
photoresponse. One photodetector (S4) works at O-band, corresponding to the responsivity at wavelengths from $1260 \mathrm{~nm}$ to $1320 \mathrm{~nm}$ (Fig. 4c), and the detailed characteristics of S4 are illustrated (Supplementary Fig. 8). Another photodetector (S1) works at wavelengths from $1485 \mathrm{~nm}$ to 1565 $\mathrm{nm}$ (Fig. 4c). The fluctuating responsivity at different wavelengths could be attributed to incident power variation at different wavelengths. During this measurement, the output power of the laser at different wavelengths was fixed up, so the practical incident power passing through the waveguide at different wavelengths was different induced by the various grating coupling efficiency. A smaller incident power usually brings about a large photocurrent gain and responsivity, which accounts for responsivity at the wavelengths with weak coupling efficiency is higher in Fig. 4c.

Noise analysis. Moreover, we analyzed noise-equivalent power $\left(N E P=i_{n} / R\right)$ of the $\mathrm{PdSe}_{2}$ photodetectors, where $i_{n}$ is the noise current which mainly results from $1 / \mathrm{f}$ noise, shot noise, and thermal noise. The measured 1/f noise spectral density in Fig. 4d indicated that $\mathrm{PdSe}_{2}$ photodetectors possess typical characteristics of 1/f noise, which can be determined by the Hooge's empirical relationship $\left(S_{i}=A \cdot i^{\alpha} / f^{\beta}\right),{ }^{61,62}$ where $\mathrm{i}, \mathrm{f}, \mathrm{A}$ are the channel current, the frequency, and noise amplitude, respectively. Current noise induced by $1 / \mathrm{f}$ noise decreases gradually with the increasing applied frequency. When photodetectors work at a high frequency, shot noise and thermal noise are dominant, which is little influenced by the applied frequency. ${ }^{62,63}$ Current noise induced by shot noise is determined by dark current $\left(i_{d}\right)$, and the measured shot noise is $1.21 \times 10^{-23} \mathrm{~A}^{2} \cdot \mathrm{Hz}^{-1}$ at $-5 \mathrm{~V}$. Additionally, the thermal noise is evaluated to be $1.25 \times 10^{-25} \mathrm{~A}^{2} \cdot \mathrm{Hz}^{-1}$ by $S_{t}=4 k \cdot T \cdot \Delta f / R_{S}$ at -5 $\mathrm{V}$, where $\mathrm{k}, \mathrm{t}, \mathrm{R}_{\mathrm{s}}$ are the Boltzmann constant, the temperature (about $300 \mathrm{~K}$ in our experiment), and the device's resistance. Therefore, NEP is mainly decided by shot noise at a high frequency, and is estimated to be $4.0 \mathrm{pW} \cdot \mathrm{Hz}^{-0.5}$ at $-5 \mathrm{~V}$. Moreover, current noise for a $\mathrm{PdSe}_{2}$ photodetector increases with the applied voltage, but a smaller NEP is obtained at the larger bias voltage (Fig. 5a). This phenomenon is attributed to the fact that a larger bias voltage brings about a higher efficiency of the separation-collection process of photogenerated carriers and a larger responsivity.

High-speed photoresponse. The frequency responses for $\mathrm{PdSe}_{2}$ photodetectors were characterized as well. The measurement setup is shown (Supplementary Fig. 9a), and the measured $3 \mathrm{~dB}$ bandwidths for S1, S2, and S3 are about $0.2,0.9$ and $1.5 \mathrm{GHz}$, respectively (Fig. 5b). The total response time for the proposed $\mathrm{PdSe}_{2}$ photodetector is determined by the carrier a transit time $\left(\tau_{t r}\right)$, 
charge/discharge time of the junction capacitance $\left(\tau_{R C}\right)$. Thr $\tau_{t r}$ can be written as $\tau_{t r}=\frac{l^{2}}{2 \mu V_{D S}}$, where $1, \mu, V_{D S}$ are the length a $\mathrm{PdSe}_{2}$ photodetector, mobility of a $\mathrm{PdSe}_{2}$ flake, and bias voltage, respectively, which is negatively correlated as the bias voltage. The measured $3 \mathrm{~dB}$ bandwidths kept almost at the same value for S3 (Supplementary Fig. 10), indicating that total response time $(\tau)$ is determined by $\tau_{R C}$. Impedance matching in measurement is necessary for a $\mathrm{PdSe}_{2}$ photodetector with larger bandwidth in the future. Furthermore, decreasing channel length and shortening transit time $\mathrm{e}^{31}$ promote obtaining an even larger bandwidth. Additionally, we performed an eye-diagram measurement to illustrate the feasibility of $\mathrm{PdSe}_{2}$ photodetectors for receiving high bit-rate data in high-speed optical communication. The measurement setup is shown (Supplementary Fig. 9b), and the experimental results in Fig. $5 \mathrm{c}$ demonstrate a completely open eye at $3 \mathrm{~V}$ with a data rate of 2.5 Gbit $\cdot \mathrm{s}^{-1}$. An improved eye diagram could be obtained when increasing the applied bias voltage because of the increase of the signal-to-noise ratio.

a

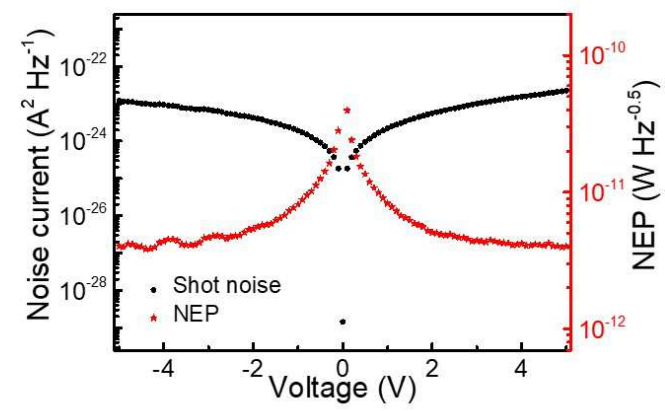

C

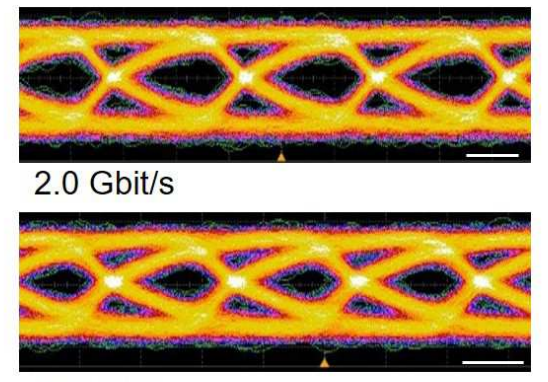

$2.5 \mathrm{Gbit} / \mathrm{s}$ b

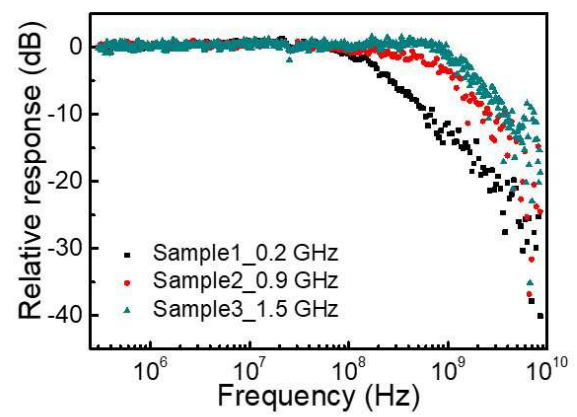

d

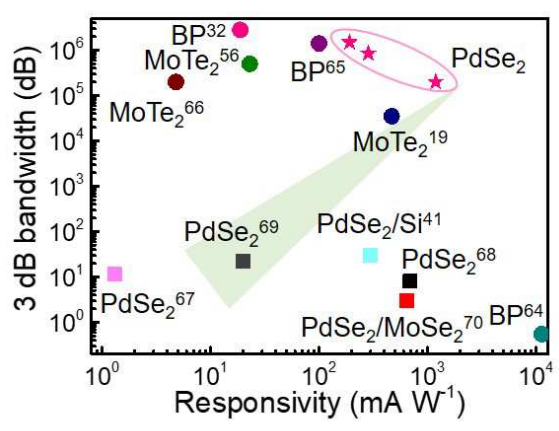

Fig. 5 Dynamic performance of PdSe 2 photodetectors. a NEP and noise current induced by shot noise at different bias voltage. b Measured frequency response of three $\mathrm{PdSe}_{2}$ photodetectors ( $\mathrm{S} 1$, $\mathrm{S} 2$ and S3) at 3V. $\mathbf{c}$ Receiver eye diagram at a data rate of 2.0 and $2.5 \mathrm{Gbit} \mathrm{s}^{-1}$ measured with a $\mathrm{PdSe}_{2}$ photodetector (S3). Scale bar: 200 ps. d Summary of the performance for waveguideintegrated photodetectors (circular shapes) based on 2D semiconductor materials and free-space 
photodetectors (rectangular shapes) based on $\mathrm{PdSe}_{2}$ flakes. Three five-pointed stars represent waveguide-integrated photodetectors (S1, S2, and S3). The green arrow indicates the direction of device development with improved performance.

\section{Discussion}

To compare with currently reported photodetectors operating in the current telecommunication bands, the responsivity and $3 \mathrm{~dB}$ bandwidth of some obtained $\mathrm{PdSe}_{2}$ photodetectors and waveguideintegrated photodetectors based on 2D semiconductor materials are listed in Fig. 5 d. ${ }^{19,32,41,56,64-70} \mathrm{On}$ the one hand, these achieved $\mathrm{PdSe}_{2}$ photodetectors possess apparent advantages such as high responsivity and broadband spectral operation, but $3 \mathrm{~dB}$ bandwidths of these $\mathrm{PdSe}_{2}$ photodetectors are smaller than $30 \mathrm{kHz}$, which limits them to high-speed applications. The waveguide-integrated $\mathrm{PdSe}_{2}$ photodetector in this work possesses a $3 \mathrm{~dB}$ bandwidth of about $1.5 \mathrm{GHz}$, which is five orders of magnitude larger than these $\mathrm{PdSe}_{2}$ photodetectors. On the other hand, waveguide-integrated photodetectors based on 2D semiconductor materials possess a small dark current, compared with the counterparts based on 2D semimetal such as graphene. A large optical bandgap of $\mathrm{MoTe}_{2}(1.04$ $\mathrm{eV}$ ) prevents it from detection at $\mathrm{C}$ band, ${ }^{56}$ and a strain-reduced bandgap allows $\mathrm{MoTe}_{2}$ to detect incident light at C-band with a 3-dB bandwidth of $35 \mathrm{MHz}$ that is not enough for some high-speed applications. ${ }^{19}$ Moreover, vertical $\mathrm{MoTe}_{2} /$ graphene heterostructure showed a large bandwidth of 50 $\mathrm{GHz}$ at O-band by shortening the transit time. ${ }^{31}$ Therefore, heterostructure photodetectors such as $\mathrm{PdSe}_{2} /$ graphene and $\mathrm{PdSe}_{2} / \mathrm{ITO}$ probably bring about a larger bandwidth at O- to C-band in the future. Additionally, BP photodetectors possess a bandwidth more than $1 \mathrm{GHz}$ at C-band, but the air-unstability leads to a complex experiment condition and performance degradation in air. Overall, the proposed $\mathrm{PdSe}_{2}$ photodetectors possess a low dark current, high responsivity from O- to C-band, large $3 \mathrm{~dB}$ bandwidth, and air stability. Therefore, a $\mathrm{PdSe}_{2}$ flake provides a new alternative to design a high-performance waveguide-integrated photodetector at current telecommunication bands even mid-infrared (MIR) regions, promoting the development of various applications of waveguideintegrated photodetectors such as on-chip spectroscopy, sensing, and optical communications.

In conclusion, we have proposed and realized integrated photodetectors based on SOI ridge waveguides and multilayer $\mathrm{PdSe}_{2}$ flakes covering the entire datacom and telecom wavelength range $(1260 \mathrm{~nm}-1565 \mathrm{~nm})$, for the first time, with both high responsivity and speed. In this work, we have demonstrated four waveguide-integrated $\mathrm{PdSe} 2$ photodetectors (S1, S2, S3, S4) whose detailed 
performance was listed in Supplementary Table 1. The obtained photodetectors possessed a broadband spectral response ranging from the O- to the C-band, and the highest responsivity (S1) was up to $1190.2 \mathrm{~mA} \cdot \mathrm{W}^{-1}$ at $5 \mathrm{~V}$ at $1550 \mathrm{~nm}$. The $3 \mathrm{~dB}$ bandwidth was about $1.5 \mathrm{GHz}$, and the eye diagram measurement indicated the $\mathrm{PdSe}_{2}$ photodetectors could be used for receiving data transmission with a data rate being more than $2.5 \mathrm{Gbit} \cdot \mathrm{s}^{-1}$. Furthermore, noise characteristics including low-frequency noise, shot noise and thermal noise were analyzed, and shot noise was the dominant noise region at high frequency, leading to a NEP of about $4.0 \mathrm{pW} \cdot \mathrm{Hz}^{-0.5}$ at $5 \mathrm{~V}$. In the future, the operational wavelengths can be extended to MIR because of the narrow bandgap of multilayer $\mathrm{PdSe}_{2}$. The $3 \mathrm{~dB}$ bandwidth can be optimized by both reducing the contact resistance between $\mathrm{PdSe}_{2}$ flakes and metal electrodes and shortening the channel length. Overall, these results pave the way for integrating $\mathrm{PdSe}_{2}$ flake onto a silicon photonic platform and provide a new route to integrated photodetectors for applications in diverse areas, including optical communications, onchip spectroscopy, and sensing.

\section{METHODS}

Device fabrication. The detailed fabrication processes are shown in Supplementary Fig. 11. Optical devices were fabricated on $220 \mathrm{~nm} \mathrm{SOI}$ wafers with a $2 \mu \mathrm{m}$-thick $\mathrm{SiO}_{2}$ box layer with multiproject wafer (MPW) processes in IMECAS. First, optical devices including grating, waveguide and MMI were patterned by deep ultra-violet (DUV) photolithography and inductive coupling plasma (ICP) etching process. Second, phosphorus ion implantation was carried out to form the lightly doped regions with a doping concentration of $5 \times 10^{17} \mathrm{~cm}^{-3}$ and the heavily doped doping regions with a doping concentration of $1.7 \times 10^{20} \mathrm{~cm}^{-3}$. Third, a thin $\mathrm{Al}_{2} \mathrm{O}_{3}$ layer of about $15 \mathrm{~nm}$ was deposited onto the prepared wafer by an Atomic Layer Deposition System (Sentech Instruments $\mathrm{GmbH}$, SI ALD). Fourth, a part of the $\mathrm{Al}_{2} \mathrm{O}_{3}$ layer (the heavily doped region) was exposed by photolithography, etched by HF, and followed with depositing Ti/Au (5/100 $\mathrm{nm})$ by electron-beam evaporation (ULvac vacuum technology (Suzhou) Co., Ltd, Ei-5Z) and a standard lift-off process. Next, source and drain electrodes were defined by electron-beam lithography (Raith, Voyager), followed by depositing Ti/Au $(5 / 100 \mathrm{~nm})$ by electron-beam evaporation and a standard lift-off process. Finally, the multilayer $\mathrm{PdSe}_{2}$ flakes were exfoliated from bulk $\mathrm{PdSe}_{2}$ crystal by Scotch tape, aligned and transferred onto the prepared devices with the assist of polydimethylsiloxane under a 
microscope.

Device measurement. Raman spectra (WITec, Alpha300R), reflection spectra (WITec, Alpha300R), SEM (Hitachi, Regulus 8230), TEM (Thermo Fisher Scientific, Talos F200X G2), femtosecond TR spectroscopy (mFemto-TR100, Time-Tech Spectra LLC) and XPS (Thermo Fisher, ESCALAB Xi+) were applied to analyze the characteristics of the exfoliated $\mathrm{PdSe}_{2}$ flakes. The thickness of $\mathrm{PdSe}_{2}$ flakes was measured by Digital Holographic Microscopy (Lyncee tec, R2014) and AFM (Oxford Instruments, Cypher ES). The cross-section of the device configuration was characterized by a focused Helium/Neon/Gallium ion microscopy (Carl Zeiss, ORION NanoFab). Electrical measurements were collected by a semiconductor analyzer (PDA, FS-Pro), and the static optoelectronic measurements were under the excitation of a tunable semiconductor laser (Santec Corporation, TSL-550) with tunable wavelength ranging from 1260 to $1620 \mathrm{~nm}$. The setups for 3 dB bandwidth characterization are shown (Supplementary Fig. 9a). The RF signal was generated by a vector network analyzer (Agilent Technologies, E5071B, Bandwidth: $300 \mathrm{kHz}$ to $8.5 \mathrm{GHz}$ ), driving an optical modulator (Lucent Technologies, 2623NA, Bandwidth: $<20 \mathrm{GHz}$ ) at $1550 \mathrm{~nm}$. The modulated optical was amplified by EDFA (MC Fiber Optics, MCEYDFA-HP-1550-33-1-1FA-T2), passing through a polarization controller, and then coupling into a waveguide by a grating coupler. The photodetector was driven by a sourcemeter (Keithley 2400), and the generated AC photocurrent was then extracted by using a bias-tee (Anritsu, G3N46, Bandwidth: $8 \mathrm{kHz}-20 \mathrm{GHz}$ ). Eventually, the AC photocurrent was received by VNA. The setups for eye diagram measurements are shown (Supplementary Fig. 9b). The RF signal driving an optical modulator was generated by a signal quality analyzer (Agilent Technologies, N4906B: up to $12.5 \mathrm{Gbit} \cdot \mathrm{s}^{-1}$ ), and the AC photocurrent extracted by a bias-tee was received by an oscilloscope (Agilent Technologies, DSO9404A, Bandwidth: <4 GHz).

\section{ACKNOWLEDGEMENTS}

The authors thank ZJU Micro-Nano Fabrication Center at Zhejiang University, Westlake Center for Micro/Nano Fabrication and Instrumentation and Service Center for Physical Sciences at Westlake University for the facility support. The research was partially supported by the National Key Research and Development Program of China (2019YFB2203003), National Natural Science Foundation of China (Grant Numbers 12104375, 62175202, 61975179 and 91950204), the start-up 
fund of Westlake University, and the Foundation of Key Laboratory of 3D Micro/Nano Fabrication and Characterization of Zhejiang Province, Westlake University

\section{Author Contributions}

L.L, H. L., and J. W. conceived the project. J. W., H. M., C. Z., M. W., Y. Y., Y. X., B. T, Y. L., B. S., J. J. and H. D. fabricated these devices. J. W., M. W., and C. S. designed the passive optical devices. J. W. performed optical and optoelectronic measurements. L.L, H. L., and J. W. analyzed the data and wrote the manuscript. All authors commented on the manuscript.

\section{Competing interests}

The authors declare no competing financial interest.

\section{Corresponding Authors}

Lan Li, Email: lilan@,westlake.edu.cn

Hongtao Lin, Email: hometown@zju.edu.cn

\section{REFERENCES}

1. Castro Neto, A.H., Guinea, F., Peres, N.M.R., Novoselov, K.S. \& Geim, A.K. The electronic properties of graphene. Rev. Mod. Phys. 81, 109-162 (2009).

2. Yang, W.H., et al. Detection of wavelength in the range from ultraviolet to near infrared light using two parallel PtSe 2 thin Si Schottky junctions. Mater. Horizons 8, 1976-1984 (2021).

3. Zou, Z.X., et al. Epitaxial synthesis of ultrathin beta- $\mathrm{In}_{2} \mathrm{Se}_{3} / \mathrm{MoS}_{2}$ heterostructures with high visible/near-infrared photoresponse. Nanoscale 12, 6480-6488 (2020).

4. Bonaccorso, F., Sun, Z., Hasan, T. \& Ferrari, A.C. Graphene photonics and optoelectronics. Nat. Photonics 4, 611-622 (2010).

5. Sun, Z., Martinez, A. \& Wang, F. Optical modulators with 2D layered materials. Nat. Photonics 10, 227-238 (2016).

6. $\mathrm{Wu}, \mathrm{J}$., et al. The interaction between quantum dots and graphene: The applications in graphene-based solar cells and photodetectors. Adv. Funct. Mater. 28, 1804712 (2018).

7. Singh, A., et al. Refractive uses of layered and two-dimensional materials for integrated photonics. ACS Photonics 7, 3270-3285 (2020).

8. Tao, L., et al. Enhancing light-matter interaction in 2D materials by optical micro/nano architectures for high-performance optoelectronic devices. InfoMat 3, 36-60 (2021).

9. Lv, L., et al. Design and tailoring of two-dimensional Schottky, PN and tunnelling junctions for electronics and optoelectronics. Nanoscale 13, 6713-6751 (2021).

10. Chen, Y., et al. Ferroelectric-tuned van der Waals heterojunction with band alignment evolution. Nat. Commun. 12, 4030 (2021).

11. Chang, T.Y., et al. Black phosphorus mid-infrared light-emitting diodes integrated with silicon photonic waveguides. Nano Lett. 20, 6824-6830 (2020). 
12. Wu, J., et al. Two-dimensional materials for integrated photonics: Recent advances and future challenges. Small Science 1, 2000053 (2021).

13. Shiramin, L.A. \& Van Thourhout, D. Graphene modulators and switches integrated on silicon and silicon nitride waveguide. IEEE J. Sel. Top. Quantum Electron. 23, 94-100 (2016).

14. Lin, H., et al. Chalcogenide glass-on-graphene photonics. Nat. Photonics 11, 798-805 (2017).

15. Liu, M., et al. A graphene-based broadband optical modulator. Nature 474, 64-67 (2011).

16. Datta, I., et al. Low-loss composite photonic platform based on 2D semiconductor monolayers. Nat. Photonics 14, 256-262 (2020).

17. Wang, X., Cheng, Z., Xu, K., Tsang, H.K. \& Xu, J.-B. High-responsivity graphene/siliconheterostructure waveguide photodetectors. Nat. Photonics 7, 888-891 (2013).

18. Schuler, S., et al. High-responsivity graphene photodetectors integrated on silicon microring resonators. Nat. Commun. 12, 3733 (2021).

19. Maiti, R., et al. Strain-engineered high-responsivity MoTe $\mathrm{M}_{2}$ photodetector for silicon photonic integrated circuits. Nat. Photonics 14, 578-584 (2020).

20. Pospischil, A., et al. CMOS-compatible graphene photodetector covering all optical communication bands. Nat. Photonics 7, 892-896 (2013).

21. Gan, X., et al. Chip-integrated ultrafast graphene photodetector with high responsivity. Nat. Photonics 7, 883-887 (2013).

22. Zheng, S.N., et al. Microring resonator-assisted Fourier transform spectrometer with enhanced resolution and large bandwidth in single chip solution. Nat. Commun. 10, 1-8 (2019).

23. Ma, Y., et al. Heterogeneously integrated graphene/silicon/halide waveguide photodetectors toward chip-scale zero-bias long-wave infrared spectroscopic sensing. ACS Nano 15, 10084-10094 (2021).

24. Zhou, X., Bec, J., Yankelevich, D. \& Marcu, L. Multispectral fluorescence lifetime imaging device with a silicon avalanche photodetector. Opt. Express 29, 20105-20120 (2021).

25. Pelucchi, E., et al. The potential and global outlook of integrated photonics for quantum technologies. Nature Reviews Physics, 1-15 (2021).

26. Marconi, S., et al. Photo thermal effect graphene detector featuring $105 \mathrm{Gbit} \mathrm{s}^{-1}$ NRZ and 120 Gbit s$^{-1}$ PAM4 direct detection. Nat. Commun. 12, 806 (2021).

27. Wang, Y., et al. Ultrahigh-speed graphene-based optical coherent receiver. Nat. Commun. 12, 5076 (2021).

28. Guo, J., et al. High-performance silicon-graphene hybrid plasmonic waveguide photodetectors beyond 1.55 um. Light-Sci. Appl. 9, 29 (2020).

29. Mišeikis, V., et al. Ultrafast, zero-bias, graphene photodetectors with polymeric gate dielectric on passive photonic waveguides. ACS Nano 14, 11190-11204 (2020).

30. Wu, J., et al. High-performance waveguide-integrated $\mathrm{Bi}_{2} \mathrm{O}_{2} \mathrm{Se}$ photodetector for $\mathrm{Si}$ photonic integrated circuits. ACS Nano 15, 15982-15991 (2021).

31. Flöry, N., et al. Waveguide-integrated van der Waals heterostructure photodetector at telecom wavelengths with high speed and high responsivity. Nat. Nanotechnol. 15, 118124 (2020).

32. Youngblood, N., Chen, C., Koester, S.J. \& Li, M. Waveguide-integrated black phosphorus photodetector with high responsivity and low dark current. Nat. Photonics 9, 247-252 
(2015).

33. Yin, Y., et al. High-speed and high-responsivity hybrid silicon/black-phosphorus waveguide photodetectors at $2 \mu \mathrm{m}$. Laser Photonics Rev. 13, 1900032 (2019).

34. Cheng, P.K., et al. Utilization of group $102 \mathrm{D}$ TMDs-PdSe ${ }_{2}$ as a nonlinear optical material for obtaining switchable laser pulse generation modes. Nanotechnology 32, 9 (2021).

35. Wu, D., et al. Fabrication of 2D PdSe $/ 3 \mathrm{D}$ CdTe mixed-dimensional van der Waals heterojunction for broadband infrared detection. ACS Appl. Mater. Inter. 13, 41791-41801 (2021).

36. Luo, L.B., et al. PdSe $e_{2}$ multilayer on germanium nanocones array with light trapping effect for sensitive infrared photodetector and image sensing application. Adv. Funct. Mater. 29, 8 (2019).

37. Sun, J., Shi, H., Siegrist, T. \& Singh, D.J. Electronic, transport, and optical properties of bulk and mono-layer PdSez. Appl. Phys. Lett. 107, 153902 (2015).

38. Oyedele, A.D., et al. PdSe: Pentagonal two-dimensional layers with high air stability for electronics. J. Am. Chem. Soc. 139, 14090-14097 (2017).

39. Chow, W.L., et al. High mobility 2D palladium diselenide field-effect transistors with tunable ambipolar characteristics. Adv. Mater. 29, 1602969 (2017).

40. Liang, F.-X., et al. Light confinement effect induced highly sensitive, self-driven nearinfrared photodetector and image sensor based on multilayer $\mathrm{PdSe}_{2} /$ pyramid $\mathrm{Si}$ heterojunction. Smal/ 15, 1903831 (2019).

41. Zeng, L.-H., et al. Controlled synthesis of 2D palladium diselenide for sensitive photodetector applications. Adv. Funct. Mater. 29, 1806878 (2019).

42. Wei, M., et al. Layer-dependent optical and dielectric properties of centimeter-scale $\mathrm{PdSe}_{2}$ films grown by chemical vapor deposition. NPJ 2D Mater. Appl. 6, 1-8 (2022).

43. Liang, Q.J., et al. High-performance, room temperature, ultra-broadband photodetectors based on air-stable PdSez. Adv. Mater. 31, 9 (2019).

44. Long, M., et al. Palladium diselenide long-wavelength infrared photodetector with high sensitivity and stability. ACS Nano 13, 2511-2519 (2019).

45. Ahmad, W., et al. Strong interlayer transition in few-layer InSe/PdSe 2 van der Waals heterostructure for near-infrared photodetection. Adv. Funct. Mater. 31, 2104143 (2021).

46. Li, Y., Chen, Y., Zhou, H. \& Zhu, H. Transient optical modulation of two-dimensional materials by excitons at ultimate proximity. ACS Nano 15, 5495-5501 (2021).

47. Ye, C., et al. Layer-tunable nonlinear optical characteristics and photocarrier dynamics of 2D PdSe $\mathrm{i}_{2}$ in broadband spectra. Small, 2103938 (2021).

48. Sun, D., et al. Ultrafast relaxation of excited Dirac fermions in epitaxial graphene using optical differential transmission spectroscopy. Phys. Rev. Lett. 101, 157402 (2008).

49. Xia, F., Mueller, T., Lin, Y.-m., Valdes-Garcia, A. \& Avouris, P. Ultrafast graphene photodetector. Nat. Nanotechnol. 4, 839-843 (2009).

50. Zhang, G., et al. Optical and electrical properties of two-dimensional palladium diselenide. Appl. Phys. Lett. 114, 253102 (2019).

51. Liu, Y., et al. Approaching the Schottky-Mott limit in van der Waals metal-semiconductor junctions. Nature 557, 696-700 (2018).

52. Xie, C., et al. Giant thickness-tunable bandgap and robust air stability of 2D palladium diselenide. Sma//16, 2000754 (2020). 
53. Li, P., et al. Penta-PdPSe: A new 2D pentagonal material with highly in-plane optical, electronic, and optoelectronic anisotropy. Adv. Mater. 33, 2102541 (2021).

54. Shiue, R.-J., et al. High-responsivity graphene-boron nitride photodetector and autocorrelator in a silicon photonic integrated circuit. Nano Lett. 15, 7288-7293 (2015).

55. Schuler, S., et al. Controlled generation of a $\mathrm{p}-\mathrm{n}$ junction in a waveguide integrated graphene photodetector. Nano Lett. 16, 7107-7112 (2016).

56. Ma, P., et al. Fast $\mathrm{MoTe}_{2}$ waveguide photodetector with high sensitivity at telecommunication wavelengths. ACS Photonics 5, 1846-1852 (2018).

57. Pi, L., et al. Highly in-plane anisotropic 2D PdSe for polarized photodetection with orientation selectivity. Adv. Funct. Mater. 31, 2006774 (2021).

58. Wu, D., et al. Mixed-dimensional $\mathrm{PdSe}_{2} / \mathrm{SiNWA}$ heterostructure based photovoltaic detectors for self-driven, broadband photodetection, infrared imaging and humidity sensing. J. Mater. Chem. A 8, 3632-3642 (2020).

59. Lopez-Sanchez, O., Lembke, D., Kayci, M., Radenovic, A. \& Kis, A. Ultrasensitive photodetectors based on monolayer MoS2. Nat. Nanotechnol. 8, 497-501 (2013).

60. Youngblood, N., Anugrah, Y., Ma, R., Koester, S.J. \& Li, M. Multifunctional graphene optical modulator and photodetector integrated on silicon waveguides. Nano Lett. 14, 27412746 (2014).

61. Chen, T., Bobbert, P.A. \& van der Wiel, W.G. $1 / f$ Noise and machine intelligence in a nonlinear dopant atom network. Small Science 1, 2000014 (2021).

62. Balandin, A.A. Low-frequency $1 / f$ noise in graphene devices. Nat. Nanotechnol. 8, 549555 (2013)

63. Li, L., et al. High-performance flexible waveguide-integrated photodetectors. Optica 5, 44-51 (2018).

64. Ma, Y., et al. High-responsivity mid-infrared black phosphorus slow light waveguide photodetector. Adv. Opt. Mater. 8, 2000337 (2020).

65. Tian, R., et al. Black phosphorus photodetector enhanced by a planar photonic crystal cavity. ACS Photonics 8, 3104-3110 (2021).

66. Bie, Y.-Q., et al. A MoTe $\mathrm{M}_{2}$-based light-emitting diode and photodetector for silicon photonic integrated circuits. Nat. Nanotechnol. 12, 1124-1129 (2017).

67. Li, G., et al. Fast photothermoelectric response in CVD-grown PdSe2 photodetectors with in-plane anisotropy. Adv. Funct. Mater. 31, 2104787 (2021).

68. Wu, D., et al. Highly polarization-sensitive, broadband, self-powered photodetector based on graphene/PdSe $/$ /germanium heterojunction. ACS Nano 13, 9907-9917 (2019).

69. Dong, Z., et al. Highly efficient, ultrabroad $\mathrm{PdSe}_{2}$ phototransistors from visible to terahertz driven by mutiphysical mechanism. ACS Nano 15, 20403-20413 (2021).

70. Zhong, J., et al. PdSe $2 / \mathrm{MoSe}_{2}$ vertical heterojunction for self-powered photodetector with high performance. Nano Research 15, 2489-2496 (2021). 


\section{Supplementary Files}

This is a list of supplementary files associated with this preprint. Click to download.

- supporting.docx 